\title{
Radiology websites: Musculoskeletal imaging websites: Part 2
}

\author{
Inna K. Indrajit \\ Department of Radiology and Imaging, INHS Asvini, Colaba, Mumbai, Maharashtra, India.
}

Correspondence: Late Dr. Inna K Indrajit, Department of Radiology and Imaging, INHS Asvini, Colaba, Mumbai - 400 005, Maharashtra, India.E-mail: rajeevsivasankar@hotmail.com

The Aug 2007 issue of IJRI featured a first round of a review of websites dealing with musculoskeletal imaging. ${ }^{[1]}$

Newer websites and other educative portals focusing on musculoskeletal imaging is reviewed herein, as Musculoskeletal Imaging Websites: Part 2.

1. Bone age Estimation using a radiograph database for comparison is available at http://bones.getthediagnosis. org/. Created by Mark Hammer and Guillermo Gonzalez, the site is a repository of radiographs of the pediatric skeleton. It has iconic links to the pediatric skeleton repository, using a grid comprising age versus region. Age is represented from birth to 15 years. Regions include bones of upper extremity (hand, wrist, elbow humerus) spine, chest, pelvis and lower extremity (femur, knee, tibia, fibula, ankle and foot).

2. An online Bone Age Estimation calculator created by Lorentz Jäntschi and Sorana Bolboacă is available at http://vl.academicdirect.ro/medical_informatics/ bone_age/v1.0/. Images of a given case is compared with online images to accurately derive the age. It uses the Tanner and Whitehouse (TW2) methods of bone age estimation.

3. Musculoskeletal Ultrasound Procedures Tutorial Page is a "website created to teach in a step-by-step fashion on how to do MSK Ultrasound guided procedures" and is available at http://mskultrasoundprocedures.com/. Shoulder procedures include therapeutic injection, arthrogram, calcific tendinosis, aspiration and lavage, acromioclavicular joint injection, biceps tendon sheath injection. Similarly specific procedures at elbow, wrist, hip, knee, ankle and foot are available here.

\begin{tabular}{|l|l|}
\hline \multicolumn{2}{|c|}{ Access this article online } \\
\hline Quick Response Code: \\
\hline
\end{tabular}

4. Ortho Bullets at Orthobullets.com is "an educational resource for orthopedic surgeons designed to improve through the communal efforts of those who use it as a learning resource". Founded by Dr Derek Moore, a practicing orthopedic spine surgeon, the website has many educative sections such as Q Bank, cases, trauma, spine, sports, pediatrics, hand, foot and ankle, pathology, basic science and anatomy. The Pathology section has very absorbing contents like Tumor Differentials at http://www.orthobullets.com/ pathology/8006/differential-groups and Tumor Staging at http://www.orthobullets.com/pathology/8001/ bone-tumor-staging-systems.

5. Orthopedics One is "the brain child of co-founders Christian Veillette and Joseph Bernstein" and available at http://www.orthopaedicsone.com/display/Viewpoint/ Home. The website rich in educative content has a useful starting point for Radiologists at http://www. orthopaedicsone.com/display/Main/Radiology. An Atlas of Musculoskeletal Oncology authored by James $\mathrm{O}$. Johnston is on offer at http://www.orthopaedicsone.com/ display/TumorLibrary/Home. Orthopedic Prostheses, Implants, Devices can be browsed region wise at http://www.orthopaedicsone.com/display/ODR/Home. Classification of a large list of bony injuries are dealt with at http://www.orthopaedicsone.com/display/Main/ Trauma + Classifications. Interestingly there is a section on Biostatistics too, available at http://www.orthopaedicsone. com/display/Main/Epidemiology + and + Biostatistics.

6. AO/OTA Classification of Fractures and Dislocations (previously known as the Müller/ AO Classification) is a standard comprehensive classification. In 1984, a group of AO surgeons led by Maurice E Müller published the "Classification of Fractures", which was the first comprehensive, systematic fracture classification system. An illustrative tool designed and edited by Chris Colton, Steve Krikler, Joseph Schatzker, Peter Trafton is available at https:// www2.aofoundation.org/wps/portal/surgery. Here interactively fractures of spine, acetabulum, femur, patella, tibia, foot, humerus, forearm and distal radius and hand are covered in details. 
7. Shoulderdoc at http://www.shoulderdoc.co.uk/index. asp is a site established in 2002 by Prof Lennard Funk. There are numerous educative sections on patient information, medical education, therapists and image gallery. One interesting portion is the section on Shoulder Classifications at http://www.shoulderdoc. co.uk/article.asp? section $=904$ created by Amol Tambe and Lennard Funk. Here illustrative classification information on AC Joint, acromion, biceps anchor/SLAP, biceps tendon, frozen shoulder, glenohumeral arthritis, labrum (instability), proximal humerus fracture, rotator cuff and subacromial impingement classifications are available.

8. Classification of Pelvic Ring Fractures by Ross K. Leighton, MD, Halifax, NS is available at http:// www.hwbf.org/ota/bfc/leig/exp.htm. There are numerous illustrative sections that cover Anterior and Posterior Lesions, Major Pelvic Ring Disruptions, Tile Classification, and Young Burgess Classification.

9. CTisUS at http://www.ctisus.org/is an educative portal administered by E. K. Fishman, MD, Department of Radiology, Johns Hopkins University. Musculoskeletal System is covered at http://www.ctisus.com/ teachingfiles/musculoskeletal/, and Pearls at http:// www.ctisus.com/learning/pearls/musculoskeletal covers nutshell abstracts from journal as regards bone tumors, dual energy, inflammatory disease, soft tissue and muscle tumors, CT techniques, trauma, vascular and wrist, to name a few.Musculoskeletal Teaching File Images at http://www.ctisus.com/teachingfiles has nearly a bank of 18,710 images.

10. From Radiology Assistant there is a section on Musculoskeletal Radiology at http://www. radiologyassistant.nl/en/p42037085786a0/ click-for-more-information.html comprising of several educative topics written by multiple authors. These include ankle fracture, bone tumor systematic approach and differential diagnosis, MRI of diabetic foot, elbow fractures, hip arthroplasty, knee meniscus/ non-meniscal pathology, shoulder MR anatomy/ Bankart lesions and Wrist carpal instability/fractures/. A useful webpage for ready illustrative educational reference is "Bone tumor in Alphabetical order" at http://www.radiologyassistant.nl/en/p527d547040466/ bone-tumor-in-alphabetical-order.html.

\section{Endpiece}

Few important Musculoskeletal Atlases are available as Musculoskeletal Anatomy atlases at http://depts. washington.edu/msatlas/, Bone Box at http://www. anatomyatlases.org/bonebox/index.shtml, Orthopedic Hardware Atlas at http://www.med.wayne.edu/ diagradiology/RSNA2003/Atlas.htm and Bone Scintigraphy Atlas in Paediatrics at http://www.medical-atlas.org/.

Musculoskeletal Eponyms are available as Musculoskeletal Eponyms: Who Are Those Guys? by Hunter TB et al. at http://pubs.rsna.org/doi/full/10.1148/radiographics. 20.3.g00ma20819, from Gentili.net at http://www.gentili. net/eponyms.htm, from Learning Radiology at http:// learningradiology.com/mobile/msk/fxswithnames.htm and from Radiopedia at http://radiopaedia.org/articles/ eponymous-fractures.

Comprehensive musculoskeletal system information is available from Radiopedia at http://radiopaedia.org/ encyclopaedia/all/musculoskeletal and from Pathology Outlines at http://pathologyoutlines.com/bone.html.

\section{Reference}

1. Indrajit IK. Web Review: Musculoskeletal imaging. Indian J Radiol Imaging 2007;17:227-8. 